\title{
Management of extracranial carotid artery aneurysm: A report of four cases
}

\author{
GANG SONG, LIQUN JIAO, YANFEI CHEN, YABING WANG, YAN MA and FENG LING \\ Department of Neurosurgery, Xuan Wu Hospital, Capital Medical University, Beijing 100053, P.R. China
}

Received July 19, 2014; Accepted May 21, 2015

DOI: $10.3892 /$ etm.2015.2606

\begin{abstract}
Extracranial carotid artery aneurysm (ECAA) is an uncommon type of peripheral artery aneurysm. The treatment of this disease requires surgical intervention. Due to the limited number of large studies, the safety and efficacy of surgical and interventional management of ECAA is not well characterized. The optimum treatment has not been clearly defined. The present study reports four cases of ECAA. The patients received end-to-end anastomosis, carotid artery stents and balloon occlusion, respectively. These four cases demonstrate that ECAA may be successfully managed using end-to-end anastomosis. Furthermore, end-to-end anastomosis is a better intervention compared with endovascular treatment as it facilitates the removal of lesions under direct observation. The advantage of open surgery is the surgical repair of end-to-end anastomosis under direct observation. However, few studies have investigated the effect of the timing of resection of an aneurysm on patient outcome and the control of bleeding remains a challenge for surgeons. Therefore, the proximal and distal ends of aneurysm should be under direct observation during surgery.
\end{abstract}

\section{Introduction}

Extracranial carotid artery aneurysm (ECAA) is an uncommon type of aneurysm, accounting for $0.4-4 \%$ of all peripheral artery aneurysms (1-3). Atherosclerosis is considered to be the primary cause of aneurysm formation. In addition, dysplastic, traumatic or infectious lesions, neck irradiation and other factors are infrequently reported (4-6). The spontaneous rupture of an ECAA is rare (7). Neurological abnormalities are the most common manifestation of an ECAA, whereas hemorrhages or nerve compression as a result of giant aneurysms are rarely observed (7). Prevention of thromboembolic events is the primary aim of ECAA treatments (8).

Correspondence to: Dr Feng Ling, Department of Neurosurgery, Xuan Wu Hospital, Capital Medical University, 45 Changchun Street, Beijing 100053, P.R. China

E-mail: ling-feng@vip.163.com

Key words: extracranial carotid artery pseudoaneurysm
Existing treatments for ECAA involve surgical procedures, such as resection with end-to-end anastomosis or grafting, internal carotid artery ligation or extracranial to intracranial bypass (8), and endovascular stenting (9). It has been reported that endovascular intervention for ECAA is an emerging alternative treatment that has yielded promising results (10). Due to the limited number of large studies, the safety and efficacy in surgical and interventional management of ECAA is unclear. The optimum treatment has not been clearly defined (11). The present study reports four cases of ECAA, with the aim of describing experience gained in the surgical and interventional management of ECAA. This study was approved by the ethic committee of Xuanwu Hospital. Written informed consent was obtained from all of the patients.

\section{Case reports}

Case 1. The patient was a 69-year-old woman that presented with a left-sided cervical mass for 9 years and complained of pain for weeks. The mass gradually increased in size for 2 years. The patient denied having any history of trauma, infection or previous surgical intervention. Physical examination revealed a pulsatile mass in the left-sided cervical region. Angiography and ultrasound examinations indicated that the mass was a pseudoaneurysm (size, $4.0 \times 3.1 \mathrm{~cm})$. The patient received a carotid artery stent; however, the surgery was unsuccessful and the pseudoaneurysm ruptured. Subsequently, two balloons (Balt Extrusion, Montmorency, France) were used to occlude the internal carotid artery and common carotid artery. The thrombus relocated to the distal end of the left middle cerebral artery, resulting in cerebral infarction.

Case 2. The patient was an 82-year-old woman that presented with left-sided cervical swelling and complained of hoarseness and dysphagia for the preceding year. The patient possessed no history of trauma, infection or any previous surgical intervention. Physical examination revealed the presence of a pulsatile mass in the left-sided cervical region, a weak pharyngeal reflex, and uvula to the right side. Angiography examination indicated that the mass was a pseudoaneurysm (size, $5.8 \times 3.7 \mathrm{~cm}$ ). Subsequently, angioplasty was performed by implanting one Wallstent 10-37 mm and two Wallstent 10-31 mm (Boston Scientific Corporation, Natick, MA, USA). However, the mass continued to increase in size gradually. After 2 months, the patient received a further angioplasty, and the implantation of two Wallgraft $10-50 \mathrm{~mm}$, one Wallgraft 

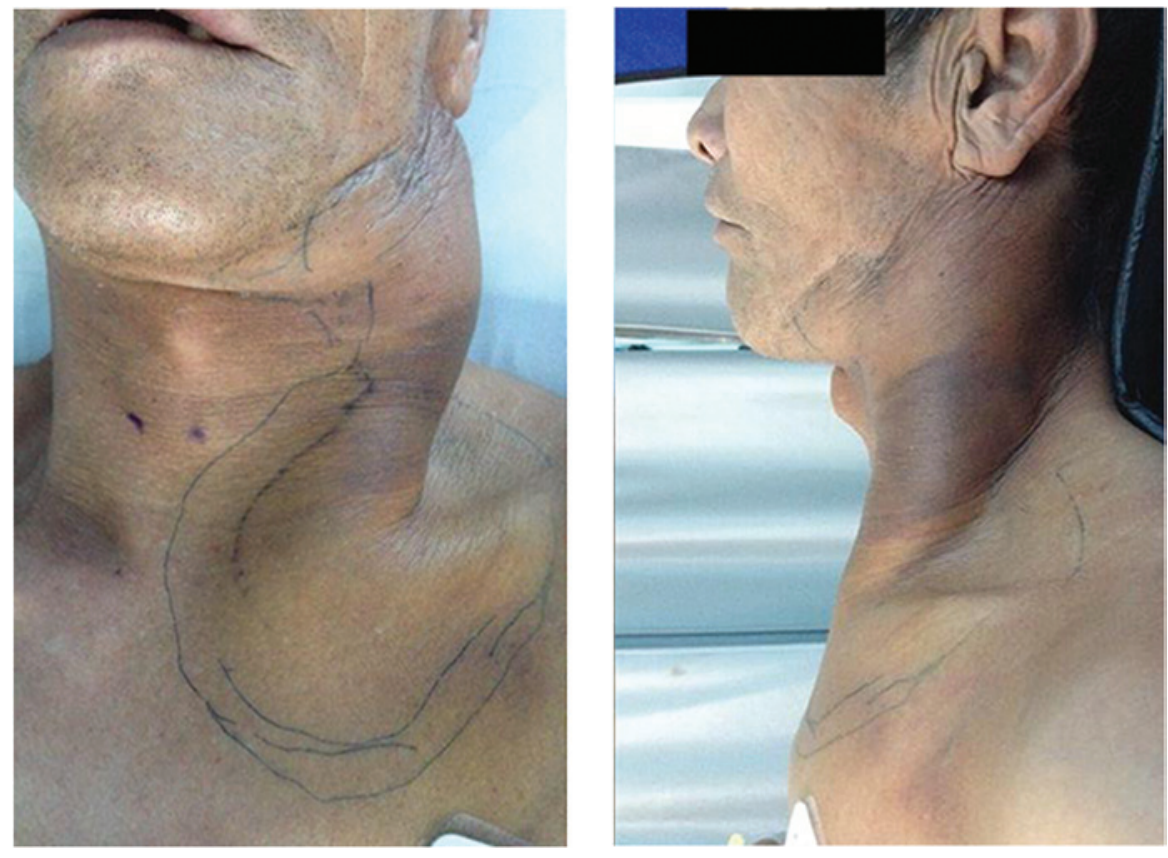

Figure 1. Case 3. Pulsatile mass was examined in the left-sided cervical region

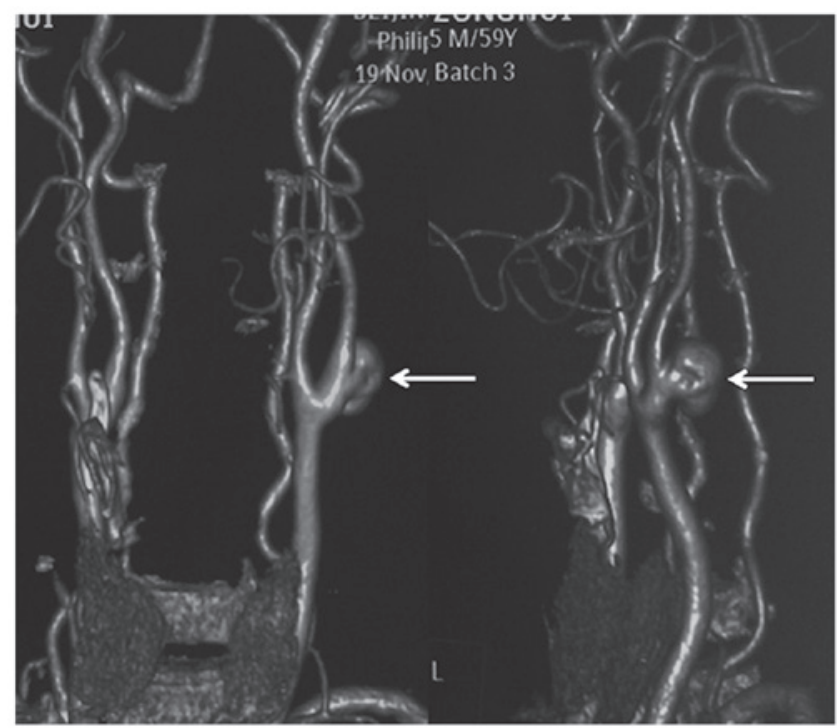

Figure 2. Case 3. Aneurysm was diagnosed by examination of the carotid artery by preoperative computed tomography angiography. White arrows indicate the extracranial carotid artery aneurysm.

8-50 $\mathrm{mm}$ and one Wallgraft 12-50 $\mathrm{mm}$ (Boston Scientific Corporation). Subsequent, ultrasound examination detected no blood flow to the pseudoaneurysm. After 2 months, the mass was ulcerated and the patient was rehospitalized for ulceration. One month subsequent to this, the patient succumbed to a skin ulcer located on the neck.

Case 3. The patient was a 59-year-old man that presented with a left-sided cervical mass and complained of hoarseness for 1 month. The patient had no history of trauma, infection or any previous surgical intervention. Physical examination revealed a pulsatile mass in the left-sided cervical region (Fig. 1). The patient was diagnosed with an aneurysm in the carotid artery using preoperative computed tomography angiography (Fig. 2). Angiography revealed that the mass was a pseudoaneurysm (size, $5.4 \times 4.6 \mathrm{~cm}$ ) and a balloon occlusion test (BOT) was negative. Two detachable balloons (Balt Extrusion) were implanted in the distal internal carotid artery, and three detachable balloons were implanted in the pseudoaneurysm. However, the mass continued to increase in size gradually. After 1 month, the patient received balloon occlusion in the proximal portion of the external carotid artery and common carotid artery (Fig. 3). Ultrasound examination indicated no blood flow into the pseudoaneurysm; 1 month later, the mass was ulcerated (Fig. 4).

Case 4. The patient was an 82-year-old woman that presented with a left-sided cervical mass and complained of hoarseness for 1 month. The patient reported no history of trauma, infection or any previous surgical intervention. Physical examination revealed a pulsatile mass in the left-sided cervical region. Angiography and ultrasound examination indicated that the mass was a pseudoaneurysm (size, $4.0 \times 2.8 \mathrm{~cm}$ ). Subsequently, a total aneurysmectomy with end-to-end oblique anastomosis was performed under general anesthesia. Subsequently, the patient made an uneventful postoperative recovery.

\section{Discussion}

Cooper reported the first treatment for ECAA by proximal ligation in 1808 (9). However, the rates of stroke and mortality for this procedure were found to be $\sim 25$ and $20 \%$, respectively (6). In 1956, the first successful resection of an aneurysm combined with reconstruction of the parent artery was conducted and accepted as the standard treatment $(12,13)$. Subsequently, the rates of mortality and neurological incidence reduced to $2.2-6.0 \%$ and $5.5-10 \%$, respectively $(1,11,14)$.

To date, the ideal method of open surgery or endovascular stenting for ECAA has not been identified. Open surgery is 

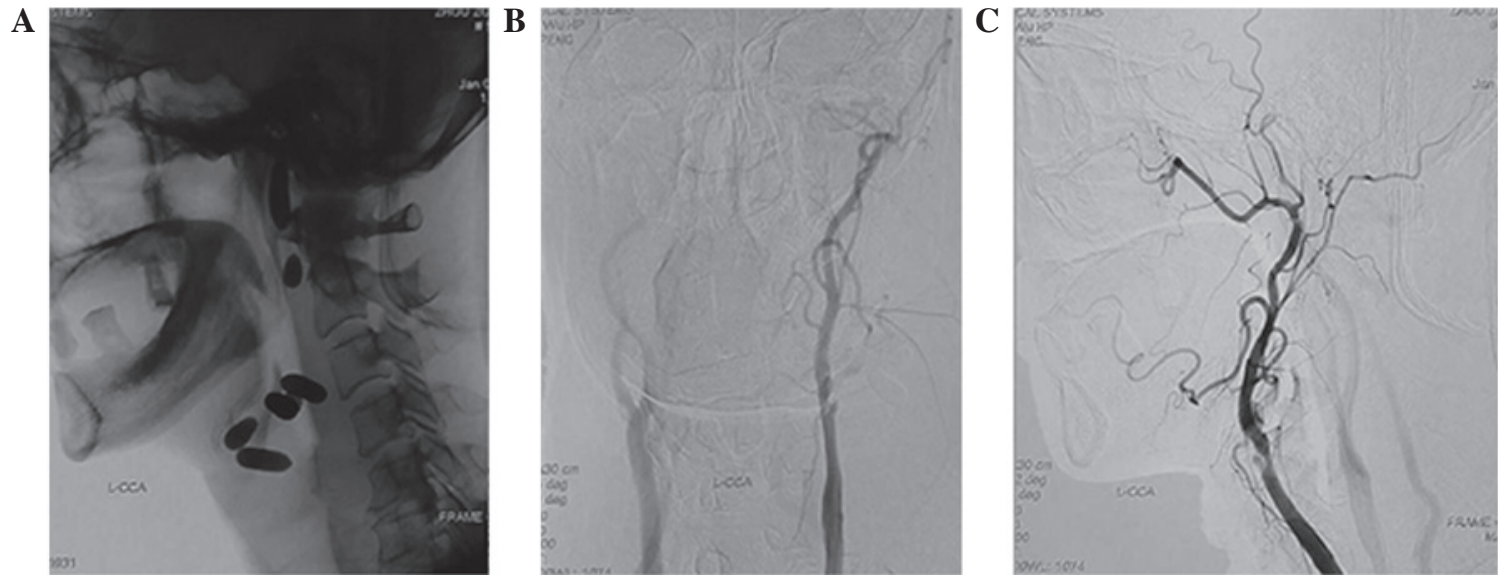

Figure 3. Case 3. (A) Balloon occlusion position. (B) Anteposterior and (C) lateral imaging of the left external carotid artery and left common carotid arterery at 1 month after treatment.

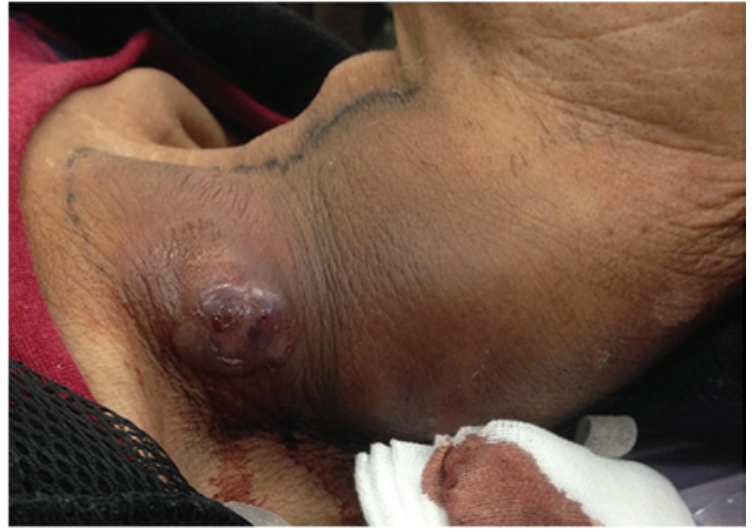

Figure 4. Case 3. Ulceration at 1 month after balloon occlusion.

generally accepted to be the preferred method of treatment for an extracranial carotid aneurysm, in order to prevent thromboembolic events. However, it is considered to have a high co-morbidity of cranial nerve deficit, of $2.2-44 \%(7,14)$. Therefore, numerous studies have investigated the use of less invasive therapies, including coil embolization, implantation of uncovered stents and stent-grafts, and have advocated the use of endovascular therapy as an alternative treatment (15-17). Li et al (18) reviewed 224 patients and observed that the rate of procedure success was $92.8 \%$, the incidence of stroke was $1.8 \%$ and in-hospital mortality was $4.1 \%$. Li et al concluded that endovascular stenting was technically feasible, with high procedure success and a relatively low complication rate in patients with ECAA. Endovascular stenting is minimally invasive, thus avoiding general anesthesia and cranial nerve deficit. Furthermore, the treatment is not limited by the location of the aneurysm, as surgical interventions may be. However, endovascular stenting is associated with a high rate of complications, including early thrombosis, late stenosis and the occlusion of the stent graft. For example, Li et al (18) reported that occlusion occurred in $6.3 \%$ of the examined patients.

Occlusion of the carotid artery using ligation results in high morbidity and mortality during follow-up (6). In the presently described cases, 3 patients underwent occlusion with detachable balloons. One patient had cerebral infarction, which led to hemiplegia. Two patients developed ulcers on the skin of the neck, and one of these patients succumbed to this condition. One patient was treated by surgical repair with end-to-end anastomosis.

Each type of treatment exhibits advantages and disadvantages. The endovascular intervention consists of carotid artery stenting and balloon occlusion. The advantage of carotid artery stenting is that it facilitates the maintenance of blood flow. However, the clinical cure rate associated with this treatment is low, which may lead to subsequent difficulty in resecting the aneurysm. Furthermore, this method is not able to restore nervous system function. For example, the case 2 patient was implanted with 7 stents. Unfortunately, the aneurysm retained blood flow. Prior to balloon occlusion, the results of a BOT should be negative. Few studies have investigated the effect of the timing of resection of an aneurysm on patient outcome, immediately resect it or after vessel wall and balloon growing together. The advantage of open surgery is that the surgical repair with end-to-end anastomosis is conducted under direct observation. However, the control of bleeding remains a challenge for surgeons. Therefore, the proximal and distal ends of aneurysm require direct observation.

\section{Acknowledgements}

This study was supported by a grant from the National 12th Five-Year Science and Technology Support Plan Project (no. 2011BAI08B00).

\section{References}

1. El-Sabrout R and Cooley DA: Extracranial carotid artery aneurysms: Texas Heart Institute experience. J Vasc Surg 31: 702-712, 2000.

2. McCollum CH, Wheeler WG, Noon GP and DeBakey ME: Aneurysms of the extracranial carotid artery. Twenty-one years' experience. Am J Surg 137: 196-200, 1979.

3. Welling RE, Taha A, Goel T, Cranley J, Krause R, Hafner C and Tew J: Extracranial carotid artery aneurysms. Surgery 93: 319-323, 1983.

4. Tabata M, Kitagawa T, Saito T, Uozaki H, Oshiro J, Miyata T and Shigematsu H: Extracranial carotid aneurysm in Takayasu's arteritis. J Vasc Surg 34: 739-742, 2001.

5. Smith BL, Munschauer CE, Diamond N and Rivera F: Ruptured internal carotid aneurysm resulting from neurofibromatosis: Treatment with intraluminal stent graft. J Vasc Surg 32: 824-8, 2000. 
6. McCann RL: Basic data related to peripheral artery aneurysms. Ann Vasc Surg 4: 411-414, 1990.

7. Rosset E, Albertini JN, Magnan PE, Ede B, Thomassin JM and Branchereau A: Surgical treatment of extracranial internal carotid artery aneurysms. J Vasc Surg 31: 713-723, 2000.

8. Szopinski P, Ciostek P, Kielar M, Myrcha P, Pleban E and Noszczyk W: A series of 15 patients with extracranial carotid artery aneurysms: Surgical and endovascular treatment. Eur J Vasc Endovasc Surg 29: 256-261, 2005.

9. Cooper A: Account of the first successful operation performed on the common carotid artery for aneurysm, in the year 1808, with the post-mortem examination in the year 1821. Guys Hosp Rep 1: 53-59, 1836.

10. Zhou W, Lin PH, Bush RL, Peden E, Guerrero MA, Terramani T, Lubbe DF, Nguyen L and Lumdsen AB: Carotid artery aneurysm: Evolution of management over two decades. J Vasc Surg 43 : 493-497, 2006.

11. Srivastava SD, Eagleton MJ, O'Hara P, Kashyap VS, Sarac T and Clair D: Surgical repair of carotid artery aneurysms: A 10-year, single-center experience. Ann Vasc Surg 24: 100-105, 2010.

12. Dimtza A: Aneurysms of the carotid arteries; report of two cases. Angiology 7: 218-227, 1956.
13. Longo GM and Kibbe MR: Aneurysms of the carotid artery. Semin Vasc Surg 18: 178-183, 2005.

14. Radak D, Davidović L, Vukobratov V, Ilijevski N, Kostić D, Maksimović Z, Vucurević G, Cvetkovic S and Avramov S: Carotid artery aneurysms: Serbian multicentric study. Ann Vasc Surg 21: 23-29, 2007.

15. Patel JV, Rossbach MM, Cleveland TJ, Gaines PA and Beard JD: Endovascular stent-graft repair of traumatic carotid artery pseudoaneurysm. Clin Radiol 57: 308-311, 2002.

16. Klein GE, Szolar DH, Raith J, Frühwirth H, Pascher O and Hausegger KA: Posttraumatic extracranial aneurysm of the internal carotid artery: Combined endovascular treatment with coils and stents. AJNR Am J Neuroradiol 18: 1261-1264, 1997.

17. Smith BL, Munschauer CE, Diamond N and Rivera F: Ruptured internal carotid aneurysm resulting from neurofibromatosis: Treatment with intraluminal stent graft. J Vasc Surg 32: 824-828, 2000

18. Li Z, Chang G, Yao C, Guo L, Liu Y, Wang M, Liu D and Wang S: Endovascular stenting of extracranial carotid artery aneurysm: A systematic review. Eur J Vasc Endovasc Surg 42: 419-426, 2011. 